\title{
Editorial
}

\section{Recent Advances in Mathematical Aspects of Engineering}

\author{
Rahmat Ellahi ${ }^{1,2, *(\mathbb{D})}$, Sadiq M. Sait ${ }^{3}(\mathbb{D})$ and Huijin $\mathrm{Xu}^{4}(\mathbb{D})$ \\ 1 Department of Mathematics \& Statistics, Faculty of Basic and Applied Sciences, \\ International Islamic University, Islamabad 44000, Pakistan \\ 2 Fulbright Fellow Department of Mechanical Engineering, University of California Riverside, \\ Riverside, CA 92521, USA \\ 3 Center for Communications and IT Research, Research Institute, \\ King Fahd University of Petroleum \& Minerals, Dhahran 31261, Saudi Arabia; sadiq@kfupm.edu.sa \\ 4 China-UK Low Carbon College, Shanghai Jiao Tong University, Shanghai 200240, China; xuhuijin@sjtu.edu.cn \\ * Correspondence: rellahi@alumni.ucr.edu
}

check for

updates

Citation: Ellahi, R.; Sait, S.M.; Xu, H. Recent Advances in Mathematical Aspects of Engineering. Symmetry 2021, 13, 811. https://doi.org/ $10.3390 /$ sym 13050811

Received: 26 January 2021

Accepted: 30 March 2021

Published: 6 May 2021

Publisher's Note: MDPI stays neutral with regard to jurisdictional claims in published maps and institutional affiliations.

\begin{abstract}
This special issue took this opportunity to invite researchers to contribute their latest original research findings, review articles, and short communications on advances in the state of the art of mathematical methods, theoretical studies, or experimental studies that extend the bounds of existing methodologies to new contributions addressing current challenges and engineering problems on "Recent Advances in Mathematical Aspects of Engineering" to be published in Symmetry.
\end{abstract}

Keywords: fluid mechanics; optimization; energy; heat transfer; steady and unsteady flow problems; porosity; nanofluids; particle shape effects; multiphase flow; thermodynamics; magnetohydrodynamics; electromagnetic; physiological fluid phenomena in biological systems; peristaltic; blood flow

\section{Introduction}

In response to a call for papers, a total of 25 papers were submitted for possible publication. After a comprehensive peer review, only 9 papers qualified for acceptance for final publication; the rest 16 papers could not be accommodated. The submissions may have been technically correct but were not considered appropriate for the scope of this special issue. The authors are from 12 geographically distributed countries: the U.S., Mexico, China, Jordan, Saudi Arabia, Pakistan, Malaysia, Vietnam, Taiwan, Thailand, Egypt, and India. This reflects the great impact of the proposed topic and the effective organization of the guest editorial team of this Special Issue. Several theoretical and experimental attempts have been devoted, and this Special Issue is one of them. We hope that this issue will not only address the current challenges but also provide an overall picture and up-to-date findings to readers of the scientific community that ultimately benefits the industrial sector regarding its specific market niches and end users.

\section{Methodologies and Usages}

The peristaltic flow of a Johnson-Segalman fluid in a symmetric curved channel with convective conditions and flexible walls is addressed in [1]. The channel walls are considered compliant. The main objective of this article is to discuss the effects of a curvilinear channel and heat/mass convection through boundary conditions. The constitutive equations for the Johnson-Segalman fluid are modeled and analyzed under the lubrication approach. The stream function, temperature, and concentration profiles are derived. The analytical solutions are obtained by using the regular perturbation method for a significant number, named the Weissenberg number. The influence of the parameter values on the physical level of interest is outlined and discussed. A comparison is made between Johnson-Segalman and Newtonian fluids. It is concluded that the axial velocity of a Johnson-Segalman fluid is substantially higher than that of a Newtonian fluid. 
Bhatti et al. [2] deal with the mass transport phenomena on particle fluid motion through an annulus. A non-Newtonian fluid propagates through a ciliated annulus in the presence of two phenomenon, namely (i) endoscopy and (ii) blood clot. The outer tube is ciliated. To examine the flow behavior, the authors consider the bi-viscosity fluid model. Mathematical modeling is formulated for a small Reynolds number to examine the inertia-free flow. The purpose of this assumption is that the wavelength-to-diameter ratio is maximal, and the pressure can be considerably uniform throughout the entire cross section. The resulting equations are analytically solved, and exact solutions are given for particleand fluid-phase profiles. The computational software Mathematica is used to evaluate both closed-form and numerical results. The graphical behavior across each parameter is discussed in detail and presented with graphs. The trapping mechanism is also shown across each parameter. It is noted clearly that the particle volume fraction and the blood clot reveal converse behavior on fluid velocity; however, the velocity of the fluid reduces significantly when the fluid behaves as a Newtonian fluid. Schmidt and Soret numbers enhance the concentration mechanism. Furthermore, more pressure is required to pass the fluid when the blood clot appears.

In [3], the problem of finite-time control for nonlinear systems with time-varying delay and exogenous disturbance is studied. First, by constructing a novel augmented Lyapunov-Krasovskii functional involving several symmetric positive definite matrices, a new delay-dependent finite-time boundedness criterion is established for the considered T-S fuzzy time-delay system by employing an improved reciprocally convex combination inequality. Then, a memory state feedback controller is designed to guarantee the finitetime boundness of the closed-loop T-S fuzzy time-delay system, which is in the framework of linear matrix inequalities (LMIs). Finally, the effectiveness and merits of the proposed results are shown by a numerical example.

The maldistribution of fluid flow through multi-channels is a critical issue encountered in many areas, such as multi-channel heat exchangers, electronic device cooling, refrigeration and cryogenic devices, air separation, and the petrochemical industry. The uniformity of flow distribution in a printed circuit heat exchanger (PCHE) is investigated in [4]. The flow distribution and resistance characteristics of a PCHE plate are studied with numerical models under different flow distribution cases. The results show that a sudden change in the angle of the fluid at the inlet of the channel can be greatly reduced by using a spreader plate with an equal inner and outer radius. The flow separation of the fluid at the inlet of the channel can also be weakened, and the imbalance of flow distribution in the channel can be reduced. Therefore, flow uniformity can be improved and the pressure loss between the inlet and outlet of PCHEs can be reduced. The flow maldistribution in each PCHE channel can be reduced to $\pm 0.2 \%$, and the average flow maldistribution in all PCHE channels can be reduced to less than $5 \%$ when the number of manifolds reaches nine. The numerical simulation of fluid flow distribution can provide guidance for subsequent research and the design and development of multi-channel heat exchangers. In summary, the symmetry of fluid flow in multi-channels for a PCHE is analyzed in this work. This work presents the frequently encountered problem of maldistribution of fluid flow in engineering, and the performance promotion leads to symmetrical aspects in both the structure and the physical process.

The entropy generation on the asymmetric peristaltic propulsion of a non-Newtonian fluid with convective boundary conditions is presented in [5]. The Williamson fluid model is considered for the analysis of flow properties. The current fluid model has the ability to reveal Newtonian and non-Newtonian behavior. The present model is formulated via momentum, entropy, and energy equations, under the approximation of a small Reynolds number and a long wavelength of the peristaltic wave. A regular perturbation scheme is employed to obtain the series solutions up to third-order approximation. All the leading parameters are discussed with the help of graphs for entropy and temperature profiles. The irreversibility process is also discussed with the help of a Bejan number. Streamlines are plotted to examine the trapping phenomena. Results obtained provide an excellent 
benchmark for further study of the entropy production with mass transfer and a peristaltic pumping mechanism.

In [6], the author examines the unsteady flow over a rotating stretchable disk with deceleration. The highly nonlinear partial differential equations of viscous fluid are simplified by existing similarity transformation. Reduced nonlinear ordinary differential equations are solved by the homotopy analysis method (HAM). The convergence of HAM solutions is also obtained. A comparison table between analytical solutions and numerical solutions is also presented. Finally, the results for useful parameters, i.e., disk stretching parameters and unsteadiness parameters, are found.

The aim of [7] is to examine the rheological significance of a Maxwell fluid configured between two isothermal stretching disks. The energy equation is extended by evaluating the heat source and sink features. The governing partial differential equations (PDEs) are converted to ordinary differential equations (ODEs) by using appropriate variables. An analytically based technique is adopted to compute the series solution of the dimensionless flow problem. The convergence of this series solution is carefully ensured. The physical interpretation of important physical parameters like the Hartmann number, Prandtl number, Archimedes number, Eckert number, heat source/sink parameter, and activation energy parameter are presented for velocity, pressure, and temperature profiles. The numerical values of different involved parameters for the skin friction coefficient and the local Nusselt number are expressed in tabular and graphical form. Moreover, the significance of an important parameter, namely Frank-Kamenetskii, is presented in both tabular and graphical form. This particular study reveals that both axial and radial velocity components decrease by increasing the Frank-Kamenetskii number and stretching the ratio parameter. The pressure distribution is enhanced with an increasing Frank-Kamenetskii number and a stretching ratio parameter. It is also observed that the temperature distribution increases with an increasing Hartmann number, Eckert number, and Archimedes number.

The key objective of the study reported in [8] is to probe the impacts of Brownian motion and thermophoresis diffusion on Casson nanofluid boundary layer flow over a nonlinear inclined stretching sheet, with the effect of convective boundaries and thermal radiations. Nonlinear ordinary differential equations are obtained from governing nonlinear partial differential equations by using compatible similarity transformations. The quantities associated with engineering aspects, such as skin friction, Sherwood number, and heat exchange, along with various impacts of material factors on the momentum, temperature, and concentration, are elucidated and clarified with diagrams. The numerical solution of the present study is obtained via the Keller-box technique and in limiting sense is reduced to the published results for accuracy purpose.

The effects of magnetohydrodynamic 3D nanofluid flow due to a rotating disk subject to Arrhenius activation energy and heat generation/absorption is examined in [9]. Flow is created due to a rotating disk. Velocity, temperature, and concentration slips at the surface of the rotating disk are considered. Effects of thermophoresis and Brownian motion are also accounted. The nonlinear expressions are deduced by the transformation procedure. The shooting technique is used to construct the numerical solution of the governing system. Plots are organized just to investigate how yjr velocity, temperature, and concentration are influenced by various emerging flow parameters. Skin-friction local Nusselt and Sherwood numbers are also plotted and analyzed. In addition, a symmetry is noticed for both components of velocity when the Hartman number increases.

\section{Future Trends in Fluid Mechanics}

The material that advances the state-of-the-art experimental, numerical, and theoretical methodologies or extends the bounds of existing methodologies through new contributions in symmetry is still insufficient, even with the completion of this Special Issue. The rheological characteristics with thin films under the influence of different nanoparticles and shapes can help with the development of better applications in industry. 
Author Contributions: Conceptualization, R.E.; S.M.S.; writing—original draft preparation; formal analysis, H.X. All authors have read and agreed to the published version of the manuscript.

Funding: This research received no external funding.

Institutional Review Board Statement: Not applicable.

Informed Consent Statement: Not applicable.

Data Availability Statement: Not applicable.

Acknowledgments: The guest editorial team of Symmetry would like to thank all authors for contributing their original work to this special issue, no matter what the final decision on their submitted manuscript was. The editorial team would also like to thank all anonymous professional reviewers for their valuable time, comments, and suggestions during the review process. We also acknowledge the entire staff of the journal's editorial board for providing their cooperation regarding this Special Issue. We hope that this issue will not only provide an overall picture and most up-to-date findings to readers from the scientific community working in the field but also benefit the industrial sectors in specific market niches and end users.

Conflicts of Interest: The author declares no conflict of interest.

\section{References}

1. Yasmin, H.; Iqbal, N.; Hussain, A. Convective Heat/Mass Transfer Analysis on Johnson-Segalman Fluid in a Symmetric Curved Channel with Peristalsis: Engineering Applications. Symmetry 2020, 12, 1475. [CrossRef]

2. Bhatti, M.M.; Elelamy, A.F.; Sait, S.M.; Ellahi, R. Hydrodynamics Interactions of Metachronal Waves on Particulate-Liquid Motion through a Ciliated Annulus: Application of Bio-Engineering in Blood Clotting and Endoscopy. Symmetry 2020, 12, 532. [CrossRef]

3. Ruan, Y.; Huang, T. Finite-Time Control for Nonlinear Systems with Time-Varying Delay and Exogenous Disturbance. Symmetry 2020, 12, 447. [CrossRef]

4. Ke, H.; Lin, Y.; Ke, Z.; Xiao, Q.; Wei, Z.; Chen, K.; Xu, H. Analysis Exploring the Uniformity of Flow Distribution in Multi-Channels for the Application of Printed Circuit Heat Exchangers. Symmetry 2020, 12, 314. [CrossRef]

5. Riaz, A.; Bhatti, M.M.; Ellahi, R.; Zeeshan, A.; Sait, S.M. Mathematical Analysis on an Asymmetrical Wavy Motion of Blood under the Influence Entropy Generation with Convective Boundary Conditions. Symmetry 2020, 12, 102. [CrossRef]

6. Sadiq, M.A. Serious Solutions for Unsteady Axisymmetric Flow over a Rotating Stretchable Disk with Deceleration. Symmetry 2020, 12, 96. [CrossRef]

7. Khan, N.; Nabwey, H.A.; Hashmi, M.S.; Khan, S.U.; Tlili, I. A Theoretical Analysis for Mixed Convection Flow of Maxwell Fluid between Two Infinite Isothermal Stretching Disks with Heat Source/Sink. Symmetry 2019, 12, 62. [CrossRef]

8. Rafique, K.; Anwar, M.I.; Misiran, M.; Khan, I.; Alharbi, S.O.; Thounthong, P.; Nisar, K.S. Keller-Box Analysis of Buongiorno Model with Brownian and Thermophoretic Diffusion for Casson Nanofluid over an Inclined Surface. Symmetry 2019, 11, 1370. [CrossRef]

9. Asma, M.; Othman, W.A.M.; Muhammad, T.; Mallawi, F.; Wong, B. Numerical Study for Magnetohydrodynamic Flow of Nanofluid Due to a Rotating Disk with Binary Chemical Reaction and Arrhenius Activation Energy. Symmetry 2019, 11, 1282. [CrossRef] 